\title{
HPM method applied to solve the model of calcium stimulated, calcium release mechanism
}

\author{
H. Vazquez-Leal ${ }^{1,}$, , L. Hernandez-Martinez ${ }^{2}$, Y. Khan ${ }^{3}$, V.M. Jimenez-Fernandez ${ }^{1}$, \\ U. Filbello-Nino ${ }^{1}$, A. Diaz-Sanchez ${ }^{2}$, A.L. Herrera-May ${ }^{4}$, R. Castaneda-Sheissa ${ }^{1}$, \\ A. Marin-Hernandez ${ }^{5}$, F. Rabago-Bernal ${ }^{6}$, J. Huerta-Chua ${ }^{7}$, S.F. Hernandez-Machuca ${ }^{1}$ \\ ${ }^{1}$ Electronic Instrumentation, Universidad Veracruzana, Xalapa, Veracruz, Mexico \\ ${ }^{2}$ National Institute for Astrophysics, Optics and Electronics, Sta. Maria Tonantzintla, Puebla, Mexico \\ ${ }^{3}$ Department of Mathematics, Zhejiang University, Hangzhou, China \\ ${ }^{4}$ Micro and NanotechnologyResearch Center, Universidad Veracruzana, Boca del Rio, Veracruz, Mexico \\ ${ }^{5}$ Department of Artificial Intelligence, Universidad Veracruzana, Xalapa, Veracruz, Mexico \\ ${ }^{6}$ Institute of Physics, AutonomousUniversity of San Luis Potosi, San Luis Potosi, SLP, Mexico \\ ${ }^{7}$ Civil EngineeringSchool, Universidad Veracruzana, Poza Rica, Veracruz, Mexico
}

\section{Email address:}

hvazquez@uv.mx (H. Vazquez-Leal), luish@inaoep.mx (L. Hernandez-Martinez), yasir123@zju.edu.cn (Y. Khan), vicjimenez@uv.mx (V.M. Jimenez-Fernandez), ufilobello@uv.mx (U. Filbello-Nino), adiazsan@inaoep.mx (A. Diaz-Sanchez), leherrera@uv.mx (A.L. Herrera-May), rocastaneda@uv.mx (R. Castaneda-Sheissa), amarin@uv.mx (A. Marin-Hernandez), rabago@dec1.ifisica.uaslp.mx (F. Rabago-Bernal),jeshuerta@uv.mx (J. Huerta-Chua), shernandez@uv.mx (S.F. Hernandez-Machuca)

\section{To cite this article:}

H. Vazquez-Leal, L. Hernandez-Martinez, Y. Khan, V.M. Jimenez-Fernandez, U. Filbello-Nino, A. Diaz-Sanchez, A.L. Herrera-May, R. Castaneda-Sheissa,A. Marin-Hernandez, F. Rabago-Bernal, J. Huerta-Chua, S.F. Hernandez-Machuca. HPM Method Applied to Solve the Model of Calcium Stimulated, Calcium Release Mechanism. American Journal of Applied Mathematics. Vol. 2, No. 1, 2014, pp. $29-35$. doi: $10.11648 /$ j.ajam.20140201.15

\begin{abstract}
In this paper, homotopy perturbation method (HPM) is employed to provide an approximate, but detailed, solution for the nonlinear differential equation that describes the calcium stimulated calcium release mechanism. Comparison to the exact solutions shows that the method is extremely efficient, if initial guess is suitably chosen.
\end{abstract}

Keywords: Cellular Signaling, CICR Calcium Mechanism, Homotopy Perturbation Method

\section{Introduction}

There are a number of phenomena in biological sciences, where the precursor of a particular process is the appearance of a travelling wave of chemical concentration, mechanical deformation, electrical signals and so on [1-3].

There are, for example, both chemical and mechanical waves that propagate on the surface of many vertebrate eggs. In the case of the egg of Medaka fish, a Calcium $\left(\mathrm{Ca}^{++}\right)$wave sweeps over the surface; it emanates from the point of sperm entry. Another example, related to interacting populations, is the progressing wave of an epidemic, on which, for instance, the rabies epizootic spreading a country. Another example is the movement of microorganisms moving into a food source chemotactically directed [1].The existence of wave phenomena in biomedical sciences requires a detailed study of travelling waves, and the search for analytic solutions of the equations that govern them.

In order to show the importance of this article, we follow the reference [1] to give a brief, and elementary, introduction to the formalism of biological waves about the calcium stimulated, calcium release mechanism (CICR). This is relevant, for instance, to understand how the membrane enclosing certain fertilized amphibian eggs works.

Due to the importance of the CICR mechanism, this work proposes to implement an approximate solution with good accuracy that describes the behaviour of such process; this is done by using the homotopy perturbation method.

This paper is organized as follows. In Section 2, we introduce the CICR mechanism. In Section 3, we introduce the basic concepts of the HPM method. Section 4 will present the approximate solution for CICR mechanism. In Section 5, we summarize our findings. Finally, a brief conclusion is given in Section 6 . 


\section{Introduction to the CICR Mechanism}

During the development of living systems there is almost continual interchange of information at both inter and intra cellular level. Embryogenesis is an example on how such communication is necessary for a sequential development. Propagating waveforms of varied biochemical concentrations are the transmission medium of such information. In the developing embryo, diffusion coefficients of biological chemicals can be very small: values in the order of $10^{-9}$ to $10^{-11} \mathrm{~cm}^{2} \mathrm{sec}^{-1}$ are very common Such small diffusion coefficients imply that, to cover macroscopic distances on the order of several millimetres, it would require a very long time if diffusion is the main process involved.

Taking into account that one-dimensional diffusion equation has the form $[1,3]$

$$
\partial u / \partial t=D\left(\partial^{2} u / \partial x^{2}\right),
$$

for a chemical of concentration $u$; the time to convey information in the form of a changed concentration over a distance $L$ is $O\left(L^{2} / D\right)$. If $L$ is in the order of, say $1 \mathrm{~mm}$, typical times with the above diffusion coefficient are within the range $O\left(10^{7}\right.$ to $\left.10^{9} \mathrm{sec}\right)$, which is excessively long for most processes in early stages of embryonic development. Simple diffusion, therefore, is unlikely to be the main vehicle for transmitting information over significant distances.

In contrast to the above, when reaction kinetics and diffusion are coupled, travelling waves of chemical concentration exist and create a biochemical change much faster than diffusion processes controlled by equations like (1). This coupling gives rise to reaction diffusion equations that has the form

$$
\partial u / \partial t=D\left(\partial^{2} u / \partial x^{2}\right)+f(u)
$$

where $u$ is the concentration, $f(u)$ denotes the kinetics, and $D$ is the constant diffusion coefficient.

A biochemical switch is a mechanism whereby sufficiently large perturbation from one steady state can move a system to another steady state. An important example, which arises experimentally, is known as the calcium stimulated, calcium release mechanism. In this process, if calcium $\left(\mathrm{Ca}^{++}\right)$is perturbed above a given threshold concentration, causes the further release of sequestered calcium, that is, the system moves to another steady state. This happens, for instance, in certain calcium sites on the membrane enclosing fertilised amphibian eggs. Besides releasing calcium, such membrane is capable to recapture it. If the concentration of $\mathrm{Ca}^{++}$is denoted by $u$, it is possible to model the kinetics by the spatially homogeneous rate law, see (2).

$$
d u / d t=A(u)-r(u)+L,
$$

where $L$ represents a small leakage, $A(u)$ is the autocatalytic release of calcium, and $r(u)$ is the recapture of the released calcium. We assume that the recapture of the released calcium is governed by first order kinetics, and the autocatalytic calcium production saturates for high $\mathrm{Ca}^{++}$ [4-7]. Under those assumptions, we arrive at the reaction kinetics model equation, with the specific forms for $A(u)$ and $r(u)$, shown in the following equation

$$
d u / d t=L+\left(K_{1} u^{2} / K_{2}+u^{2}\right)-K_{3} u,
$$

where

$$
f(u)=L+\left(K_{1} u^{2} / K_{2}+u^{2}\right)-K_{3} u,
$$

here $K_{1}, K_{2}, K_{3}$, and $L$ are positive parameters.

It is possible to systematize the study of (4) considering different cases for $L$ parameter. Following, a brief description for each case is presented.

If $L=0$, there are three steady states $\left(u_{1}, u_{2}\right.$, and $\left.u_{3}\right)$ [1], two stable and one unstable [8]. As $L$ increases above certain threshold value $L_{C}, u_{1}$, and $u_{2}$ coalesce and then disappear. So, if initially $u=u_{l}$, a perturbation of $L$ large enough can result in shifting the steady state to $u_{3}$, the larger of the two stable steady states, where it will remain (see Section 4.4).

Equation (4) is a nonlinear differential equation. From the qualitative theory of this kind of equations [8], we know that equalling to zero the right side of (4) we obtain the critical points, which represent rest points in the evolution of a system and define the steady states. Nevertheless, the qualitative theory provides the asymptotic behaviour of the solution, that is, for $\mathrm{t} \rightarrow \infty$.

This work assumes that the system begins at some point $u=u(0)$, evolves, and use the homotopy perturbation method (HPM) to find, analytically, a highly accurate solution to (4). To this end, we exemplify using the following values: $K_{I}=3$, $K_{2}=1$, and $K_{3}=1$; considering cases $L=0$ ( $\left.L<L_{C}\right), L=1$ $\left(L>L_{C}\right)$, and $L=L_{C}$ with $L_{C}=0.0858683119$, besides this work will calculate an approximate version that describes the CICR switching mechanism.

\section{Homotopy Perturbation Method (HPM)}

The Homotopy Perturbation Method [9, 10] was proposed by Ji Huan He; it was introduced as a powerful tool to solve various kinds of nonlinear problems. As is well known, nonlinear phenomena appear in several scientific fields, such as applied mathematics, physics and engineering. Scientists in those disciplines constantly face the task of finding solutions to nonlinear ordinary differential equations, partial differential equations and systems of nonlinear ordinary differential equations. There are several methods available to find approximate solutions to nonlinear problems, like: variational approaches $[11,12]$, Tanh method [13], exp-function [14], Adomian's decomposition method $[15,16]$, parameter expansion [17], the HPM [9, 10, 18-24] among others.

The homotopy perturbation method could be considered as combination of classical perturbation techniques and 
homotopy (whose origin is based in topology); but has been able to reduce limitations found in traditional perturbation methods. For instance, the method does not need a small parameter or linearization, in fact, only requires less iteration to obtain highly accurate solutions. This method has been used successfully to solve integral equations, for example, the case of Volterra integral equations [22]. The method requires an initial approximation, which should contain as much information as possible about the nature of the solution. Often it can be achieved through an empirical knowledge of the solution.

To get an idea of how HPM works, consider a general nonlinear equation in the form

$$
A(u)-f(r)=0, r \in \Omega,
$$

having boundary conditions

$$
B(u, \partial u / \partial n)=0, r \in \Gamma
$$

where $A$ is a general differential operator, $B$ a boundary operator, $f(r)$ a known analytical function, and $\Gamma$ is the domain boundary $\Omega$. $A$ can be divided into two parts, $L$ and $N$; here $L$ is linear and $N$ is nonlinear. Therefore, (6) can be rewritten as

$$
L(u)+N(u)-f(r)=0 .
$$

Generally, homotopy can be constructed in the form [9]

$$
\begin{gathered}
H(v, p)=(1-p)\left[L(v)-L\left(u_{0}\right)\right]+p[A(v)-f(r)]=0, \\
p \in[0,1], r \in \Omega,
\end{gathered}
$$

or

$$
\begin{gathered}
H(v, p)=L(v)-L\left(u_{0}\right)+p L\left(u_{0}\right)+p[N(v)-f(r)]=0, \\
p \in[0,1], r \in \Omega,
\end{gathered}
$$

where $p$ is a homotopy parameter, whose values are in the range $p \in[0,1], u_{0}$ is the first approximation to the solution of (6), which satisfies the boundary conditions.

We can assume that solutions for (9) or (10) may be written as power series of $p$.

$$
v=v_{0}+v_{l} p^{l}+v_{l} p^{2}+\ldots,
$$

substituting (11) into (10) and equating terms having identical powers of $p$, we can find values for the sequence $u_{0}$, $u_{1}, u_{2}, \cdots$. When $\mathrm{p} \rightarrow 1$, results to the approximate solution of (6) may be shown as

$$
v=v_{0}+v_{1}+v_{2}+v_{3}+\cdots
$$

Another way to build a homotopy, relevant to the purpose of this paper, is considering a general equation in the form

$$
L(u)+N(u)=0,
$$

where $L(u)$ and $N(u)$ are the linear and no linear operators, respectively; so that solution for $L=0$ describes, in the best way, the original nonlinear system.

By the homotopy technique

$$
(1-p) L_{D}(v)+p(L(v)+N(v))=0,
$$

where $L_{D}$ is a linear operator employed in the homotopy method.

Again, is assumed that solution for (14) can be written in the form (11), by taking the limit when $p \rightarrow 1$, results in the approximate solution for (13).

\section{Application of HPM to Solve the Equation Calcium Stimulated, Calcium Release}

To obtain the value of $L_{C}$ we solve, simultaneously, equations $f(u)=0$ and $f^{\prime}(u)=0$, using the following values: $K_{1}=3, K_{2}=1$, and $K_{3}=1$, see (5), such that the obtained value is $L_{C}=0.0858683119$, with its corresponding critical point $u=0.1773105825$.

Equation (4) can be rewritten in the form

$$
u^{\prime}(t)+u^{2}(t) u^{\prime}(t)+u^{3}(t)-3 u^{2}(t)-L u^{2}(t)+u(t)-L=0
$$

Next, the linear and nonlinear parts are identified as

$$
\begin{gathered}
L(u)=u^{\prime}(t)+u(t)-L, \\
N(u)=u^{\prime}(t) u^{2}(t)+u^{3}(t)-3 u^{2}(t)-L u^{2}(t) .
\end{gathered}
$$

From (16) it is possible to create a linear function for the homotopy method

$$
L_{D}(u)=a u^{\prime}(t)+b u(t)+c-L,
$$

where $a, b$, and $c$ are adjustment parameters.

Substituting (11) into (14) $\left(L_{D}\right.$ is obtained from (18)) and equating terms with identical powers of $p$, we obtain

$$
\begin{gathered}
a v_{0}{ }^{\prime}(t)+b v_{0}(t)+c-L=0, \\
a v_{1}{ }^{\prime}(t)+b v_{1}(t)+\left(v_{0}^{2}(t)-a+1\right) v_{0}{ }^{\prime}(t)+v_{0}^{3}(t)- \\
(3+L) v_{0}^{2}(t)+(1-b) v_{0}(t)-c=0 .
\end{gathered}
$$

Next, solution for (19) and (20) are obtained for different cases of $L$. For all the cases the adjustment of parameters is performed employing the NonlinearFit command of Maple (release 15) software.

\subsection{Case when $L=0$}

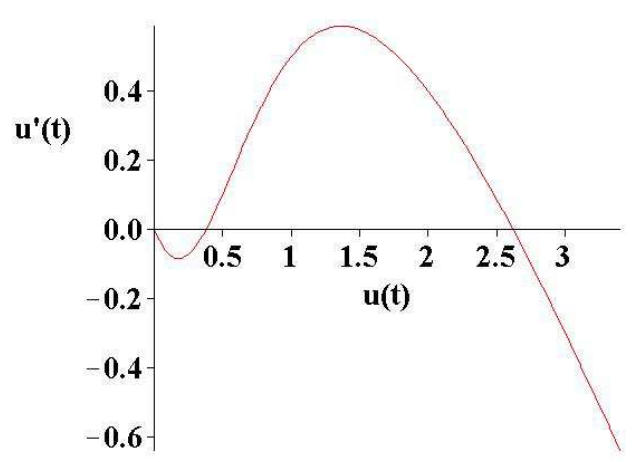

Figure 1. Phase plane for (4) when $L=0$. 
Fig. 1 shows the phase plane of (4) when $L=0$. In the same figure we see that this equation has critical points at $u=0$, $u=3 / 2-1 / 2 \sqrt{ } 5$, and $u=3 / 2+1 / 2 \sqrt{ } 5$ (those are values obtained from (5) when $f(u)=0)$.

Differential equations (19) and (20) will be solved, applying initial conditions $v_{l}(0)=0.9, v_{2}(0)=0$, and $L=0$.

First, we adjust the values of $a, b$, and $c$, so we choose the lowest order approximation.

$$
u(t)=v_{0}(t)=97 / 37-[(31 / 18) \exp (-11 t / 21)] .
$$

Fig. 2 shows the comparison between (21) and the exact solution. It can be seen that while the critical point $(3 / 2-1 / 2 \sqrt{ } 5,0)$ is unstable, the point $(3 / 2+1 / 2 \sqrt{ } 5,0)$ is stable and the system evolves asymptotically towards it [8]. It becomes clear that, even taking the lowest order of approximation, still it is possible to obtain a highly accurate solution.

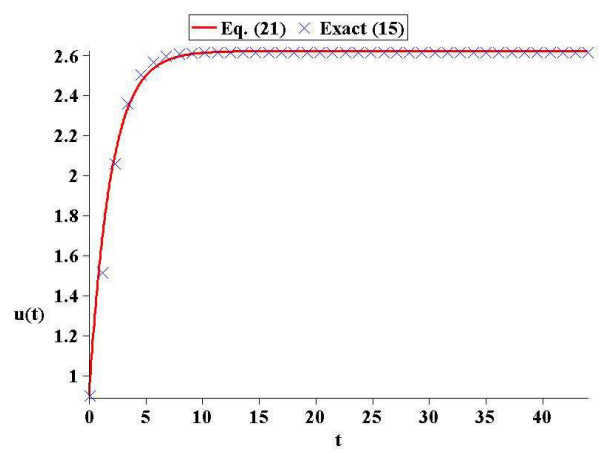

Figure 2. Approximate solution (21) and exact solution for (15).

To improve the previous approach, we adjust parameters $a$, $b$, and $c$ so that the second order approximation is chosen

$$
\begin{aligned}
u(t)= & v_{0}(t)+v_{1}(t)=(-2 \exp (-54 t / 19) / 677)-(8 \exp (-36 t / 19) / 29) \\
& +34 / 13+(-26 / 16-35 t / 24) \exp (-18 t / 19) .
\end{aligned}
$$

Now, Fig. 3 shows the comparison between (22) and the exact solution. It is evident the accuracy of (22) as an approximate solution to (4).

Next, differential equations (19) and (20) (with $L=0$ ) are solved using initial conditions $v_{l}(0)=0.2$ and $v_{2}(0)=0$.

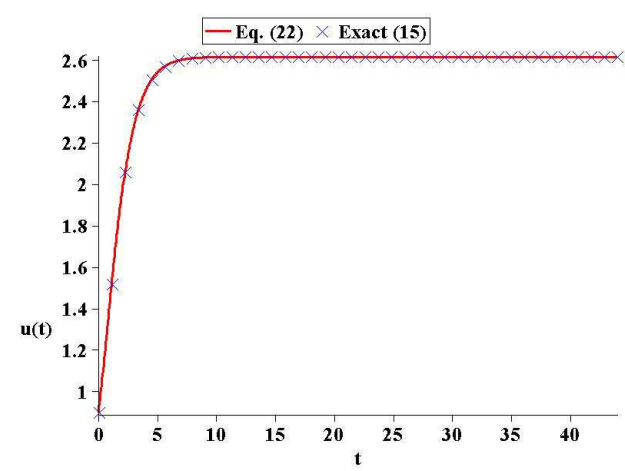

Figure 3. Approximate solution (22) and exact solution for (15) when $L=0$.

As before, values for parameters $a, b$, and $c$ are adjusted so we choose the lowest order approximation (see Fig. 4).

$$
u(t)=v_{0}(t)=(70 / 349) \exp (-37 t / 59)
$$

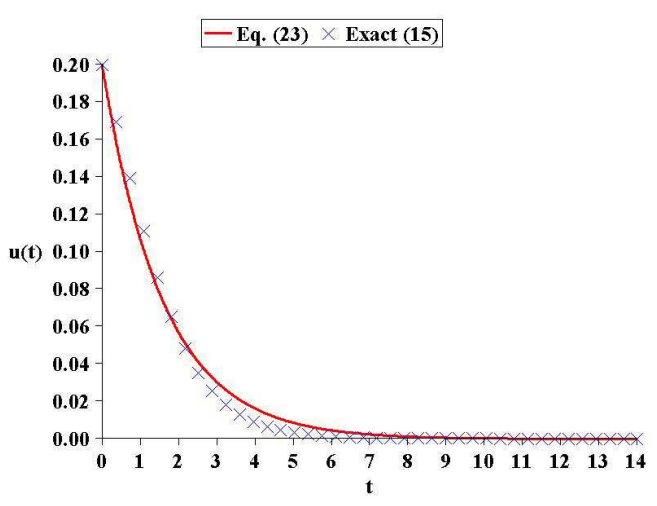

Figure 4. Approximate solution (23) and exact solution for (15) when $L=0$.

To improve the previous approach, parameters $a, b$, and $c$ are adjusted at the second order approximation

$$
\begin{gathered}
u(t)=v_{0}(t)+v_{1}(t)=(17 \exp (-133 t / 44) / 212)-(5 \exp (-133 t / 44) \\
/ 22)+(25 / 72+3 t / 67) \exp (-134 t / 133),
\end{gathered}
$$

where Fig. 5 shows the comparison between (24) and the exact solution. Point $(0,0)$ is stable and the system evolves asymptotically towards it. Therefore, (24) is a highly accurate approximation to (4).

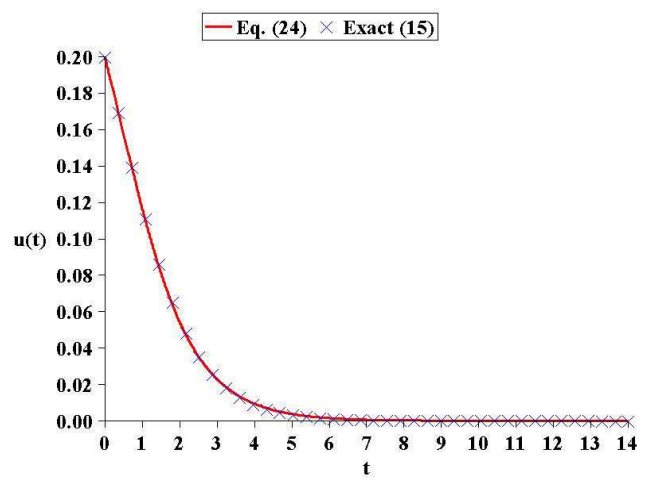

Figure 5. Approximate solution (24) and exact solution for (15) when $L=0$.

\subsection{Case when $L=L_{C}$}

Fig. 6 shows the phase plane of (4) for $L=L_{C}$. It can be seen that this equation has critical points at $u=0.1773105825$ and $u=2.731246007$.

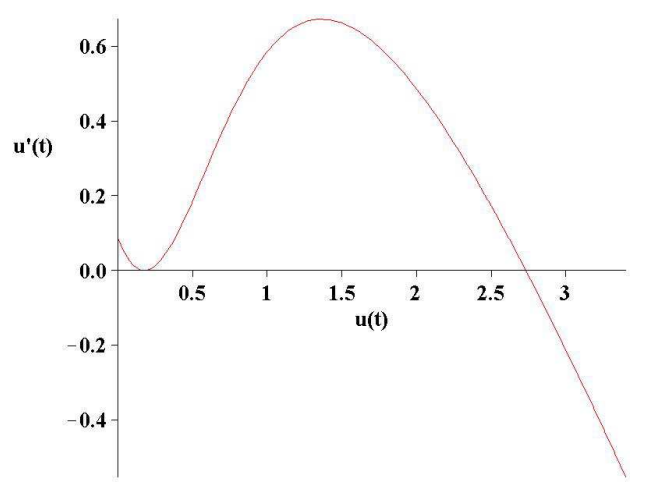

Figure 6. Phase plane for (4) when $L=L_{C}$. 
Next, (19) and (20) are solved using initial conditions $v_{1}(0)=0, v_{2}(0)=0$, and $L_{C}=0.0858683119$. For this case, parameters $a, b$, and $c$ are adjusted to choose the lowest order approximation

$$
u(t)=v_{0}(t)=13 / 80-(13 / 80) \exp (-8 t / 27),
$$

where Fig. 7 shows the comparison between (25) and the exact solution. It can be seen that the critical point $(0.1773105825,0)$ is stable and the system evolves asymptotically towards it. Therefore, even using the lowest order approximation, is possible to obtain an accurate solution.

Solving (19) subject to initial conditions $v_{l}(0)=0.9$ and $v_{2}=0$, parameters $a, b$, and $c$ are adjusted in order to choose the lowest order approximation

$$
u(t)=v_{0}(t)=52 / 19-(90 / 49) \exp (-15 t / 28)
$$

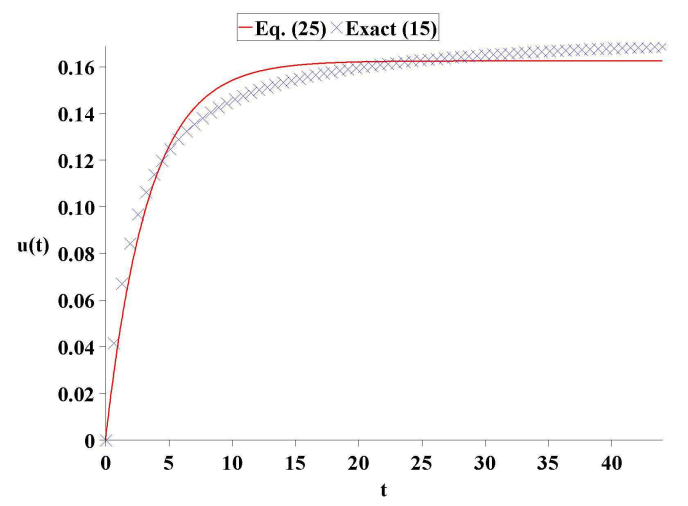

Figure 7. Approximate solution (25) and exact solution for (15) when $L=L_{C}$.

Fig. 8 shows the comparison between (26) and the exact solution. The point $(2.731246007,0)$ is stable and the system evolves asymptotically towards it. It is evident the accuracy of (26) as an approximate solution for (4).

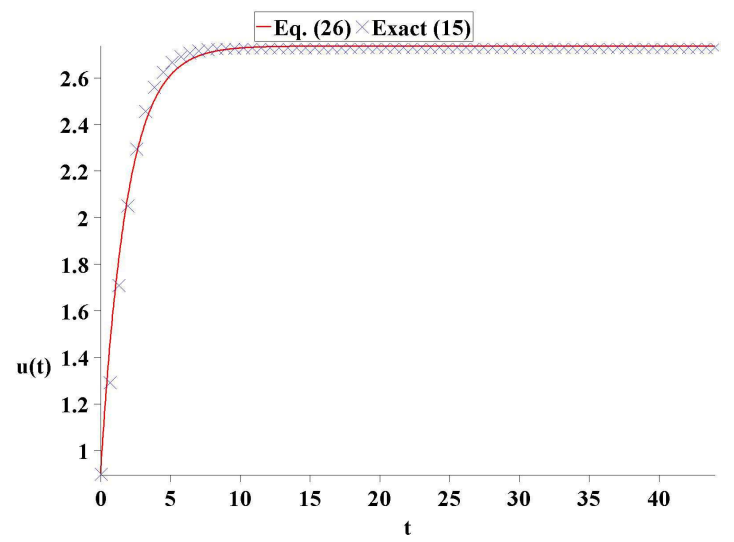

Figure 8. Approximate solution (26) and exact solution for (15) when $L=L_{C}$.

\subsection{Case when $L=1$}

Fig. 9 shows the phase plane of (4) when $L=1$. In addition, it can be seen that this equation has one critical point at $u=3.806300717$.

Solving (19) and (20) subject to initial conditions $v_{1}(0)=0.9, v_{2}(0)=0$, and $L=1$; adjusting $a, b$, and $\mathrm{c}$ the second order approximation is chosen

$$
\begin{aligned}
u(t)= & v_{0}(t)+v_{1}(t)=(-73 \exp (-86 t / 33)) / 97+(115 \exp (-33 t / 19)) \\
& / 36+53 / 14+(-293 / 55+37 t / 53) \exp (-33 t / 38) .
\end{aligned}
$$

Fig. 10 shows the comparison between (27) and the exact solution. We see that the point $(3.806300717,0)$ is stable, thus, the approach is accurate.

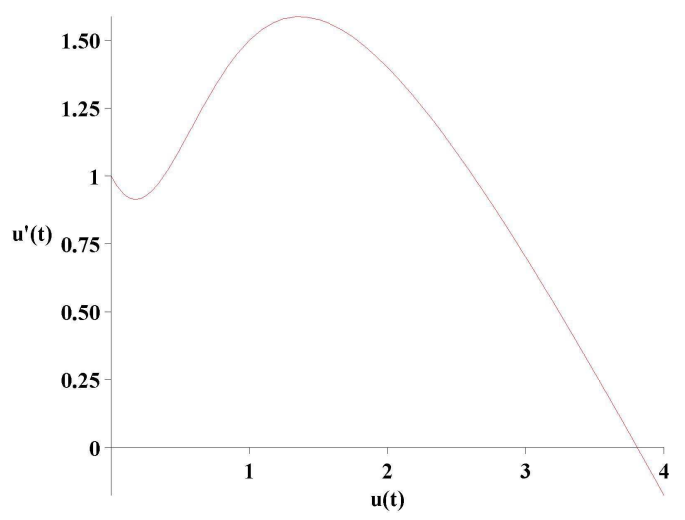

Figure 9. Phase plane for (4) when $L=1$.

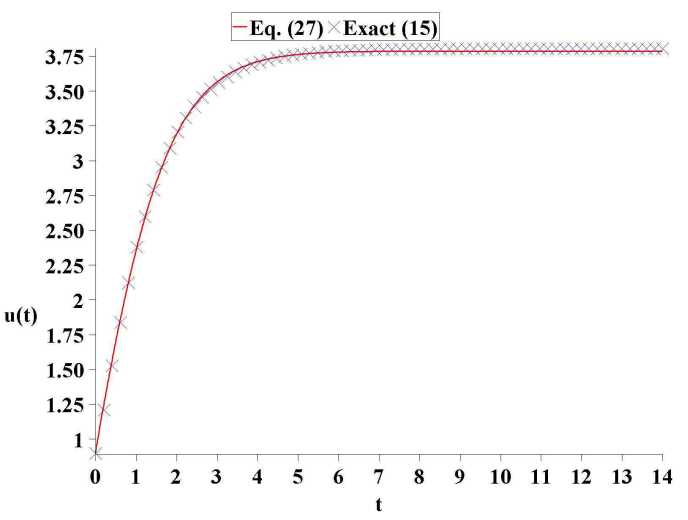

Figure 10. Approximate solution (27) and exact solution for (15) when $L=1$.

\subsection{CICR Mechanism Case}

In this section a highly accurate approximation is calculated that exemplifies a perturbation of $L$ for a steady state leading a transition towards other steady state. It means that HPM was able to successfully adapt to the different steady states depending of the perturbation of $L$. This result is the main contribution of this work to the study of CICR mechanism with semi-analytical methods.

Suppose the case where initially $L=L_{C}$, at steady state $u_{1}=0.1773105825$. Now, the state is perturbated leading to an increase from $L=L_{C}$ to $L=L_{C}+\Delta L$, where is chosen to exemplify $\Delta L$. The result is a transition from the original steady state towards a new steady state $u_{2}=3.338922$.

After applying HPM, is observed that the first approximation has good accuracy (see Fig. 11), being of the form 


$$
u(t)=v_{0}(t)=47 / 14-(47 / 14) \exp (-18 t / 37)
$$

\section{Discussion}

The nonlinear phenomena appear in several sciences fields, such as applied mathematics, physics, engineering, medicine, chemistry and biology. Scientists in those disciplines are constantly faced with the task of finding solutions for nonlinear ordinary differential equations.

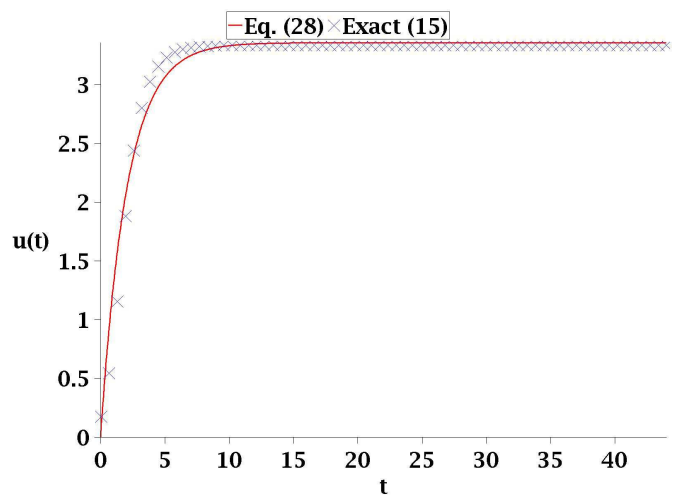

Figure 11. Approximate solution (28) and exact solution for (15).

In fact, the possibility of finding analytical solutions in those cases is very difficult. In particular, for equations like (4), the natural way to proceed is the use of techniques for qualitative theory of nonlinear differential equations; however, it is known that they provide the asymptotic behavior of the solution. In this paper, we used the homotopy perturbation method (HPM) to find a very simple and accurate solution for (4). The possibility of finding analytical expressions for quantities that describe a system is very important. In our case, we described, successfully, the detailed evolution of the concentration of $\mathrm{Ca}^{++}$, from its initial value to its asymptotic value given by stable critical points by means of analytical expressions provided in this work. At Section 4.4 we chose an initial value to exemplify the CICR mechanism. Fig. 11 showed that our approximation has good accuracy.The ultimate goal is that using the method presented in this paper, it would help to analyse in more detail the calcium stimulated, calcium released mechanism.

\section{Conclusions}

In this paper, HPM method is employed to find an analytical expression for the equation that governs the CICR mechanism. We followed in all detail the dynamical evolution in the concentration of $\mathrm{Ca}^{++}$from its initial value to its asymptotic value. This information is, clearly, very important and contrasts with the one obtained from traditional techniques using qualitative theory of nonlinear differential equations; where we know the asymptotic behavior of the solution. A relevant fact is that, depending if the trial function is suitably chosen is possible to obtain an approximation highly accurate even using the lowest order.

\section{References}

[1] J.D. Murray, "Mathematical Biology: I. An Introduction", Springer, 3rd edition, December, 2007.

[2] J. Keener, J. Sneyd, "Mathematical Physiology", Springer, October, 1998.

[3] R. Resnick, D. Halliday, "FísicaVol 1", John Wiley and Sons, Inc., 1977.

[4] G. Odell, G.F. Oster, B. Burnside, P. Alberch, "The mechanical basis for morphogenesis", Developmental Biology, vol. 85, pp. 446-462, 1981.

[5] J.D. Murray, G.F. Oster, "Cell traction models for generating pattern and form in morphogenesis", Journal of Mathematical Biology, vol. 19, num. 3, 265-279, 1986.

[6] A. Cheer, R. Nuccitelli, G.F. Oster, J.P. Vincent, "Cortical activity in vertebrate eggs I: The activation waves", Journal of Theoretical Biology, vol. 124, num. 4, pp. 377-404, 1987.

[7] D.C. Lane, J.D. Murray, V.S. Manoranjan, "Analysis of wave phenomena in a morphogenetic mechanochemical model and an application to post-fertilisation waves on eggs", IMA Journal of Mathematics Applied in Medicine and Biology", vol. 4, pp. 309-331, 1987.

[8] G.F. Simmons, "Differential equations with applications and historical notes", McGraw-Hill, $2^{\text {nd }}$ edition, 1991.

[9] J.H. He, "A coupling method of a homotopy technique and a perturbation technique for nonlinear problems" International Journal of Non-Linear Mechanics, vol. 351, pp. 37-43, 2000.

[10] J.H. He, "Homotopy perturbation technique", Computational Methods in Applied Mechanics and Engineering, vol. 178, num. 3-4, pp. 257-262, August, 1999.

[11] L.M.B. Assas, "Approximate solutions for the generalized kdv Burgers' equation by He's variational iteration method", PhysicaScripta, vol. 76, num. 2, pp. 161-164, 2007.

[12] J.H. He, "Variational approach for nonlinear oscillators", Chaos, Solitons and Fractals, vol. 34, num. 5, pp.1430-1439, 2007.

[13] D.J. Evans, K.R. Raslan, "The Tanh function method for solving some important nonlinear partial differential", International Journal of Computer Mathematics, vol. 82, pp. 897-905, 2005.

[14] F. Xu, "A generalized soliton solution of the Konopelchenko-Dubrovsky Equation using He's exp-function method", Z. Naturforsch, vol. 62a, pp. 685-688, 2007.

[15] G. Adomain, "A review of decomposition method in applied mathematics", Journal of Mathematical Analysis and Applications, vol. 135, num. 2, pp. 501-544, 1988.

[16] E. Babolian, J. Biazar, "On the order of convergence of Adomian method", Applied Mathematics and Computation, vol. 130, num. 2, pp. 383-387, 2002.

[17] L.N. Zhang, L. Xu, "Determination of the limit cycle by He's parameter expansion for oscillators in a $u^{3} / 1+u^{2}$ potential", Z. Naturforsch, vol. 62a, pp. 396-398, 2007. 
[18] J.H. He, "Homotopy perturbation method for solving boundary value problems", Physics Letters A, vol. 350, num. $1-2$, pp. 87-88, 2006.

[19] A. Fereidoon, Y. Rostamiyan, M. Akbarzade, D.D. Ganji, "Application of He's homotopy perturbation method to nonlinear shock damper dynamics", Archive of Applied Mechanics, vol. 80, num. 6, pp. 641-649, 2010.

[20] J.H. He, "Recent development of the homotopy perturbation method", Topological Methods in Nonlinear Analysis, vol. 31, num. 2, pp. 205-209, 2008.

[21] A. Beléndez, C. Pascual, M.L. Álvarez, D.I. Méndez, M.S Yebra, A. Hernández, "Higher order analytical approximate solutions to the nonlinear pendulum by He's homotopy method", PhysicaScripta, vol. 79, num. 1, pp. 1-24, 2009.
[22] J.H. He, "A coupling method of a homotopy and a perturbation technique for nonlinear problems", International Journal of Non-Linear Mechanics, vol. 35, num. 1, pp. 37-43, 2000 .

[23] M. El .Shaed, "Application of He's homotopy perturbation method to Volterra'sintegro differential equation", International Journal of Nonlinear Sciences and Numerical Simulation, vol. 6, num. 2, pp. 163-168, 2005.

[24] J.H. He, "Some Asymptotic Methods for Strongly Nonlinear Equations", International Journal of Modern Physics B, vol. 20, num. 10, pp. 1141-1199, 2006. 